\title{
Research on high-speed railway construction and the change of economic gravitation
}

\author{
Liu Zhaolu ${ }^{1}$ \\ ${ }^{1}$ Beijing Jiaotong University School of Economics and Management Beijing, China
}

\begin{abstract}
The construction of high-speed railway(HSR) can not only improve the quality of transportation infrastructure in China, but also has a significant impact on regional economy. The influence of HSR construction on economic connection intensity in the context of the coordinated development of cities in Beijing-Tianjin-Hebei region is investigated. Based on the improved Gravity Model(GM), the research shows that HSR has a positive effect on the economic gravitation, while the influence of spatial distance on economic connections of cities is negative. According to empirical analysis, it represents an obvious Coreperiphery structure after HSR is opened. It also reveals that the regional economic development is unbalanced, which is caused by the poor transportation infrastructure. In addition, the paper further offers a perspective of how to promote regional economy.
\end{abstract}

\section{Introduction}

With the rapid development of China's economy and technology, high-speed railway(HSR) has entered an era of rapid development, and its construction and development have laid a solid foundation and guarantee for the economic development of regional cities. By the end of 2018, China's high-speed railway has reached 29000 kilometers, ranking first in the world. The construction of high-speed railway not only can deepen the connection between adjacent areas, accelerate the mutual circulation of resource elements, but also improve the accessibility between cities and save cost for regional economic development. Therefore, the improvement of traffic conditions will stimulate economic growth to a certain extent, and promote the adjustment and optimization of industrial structure.

In 2014, President Xi first proposed the coordinated development of Beijing, Tianjin and Hebei as a national strategy. The Beijing-Tianjin-Hebei urban agglomeration, one of China's three major urban agglomerations, has successively opened the Beijing-Tianjin Intercity Railway, Beijing-Shanghai High-speed Railway, BeijingShijiazhuang High-speed Railway, etc. since 2008, which marks that Beijing Tianjin Hebei urban agglomerations have entered the era of high-speed rail. At the end of 2019, the HSR line connecting Beijing and Zhangjiakou with a maximum design speed of $350 \mathrm{kph}$ officially went into operation, which marks that all prefecture-level cities in the Beijing-Tianjin-Hebei region have achieved full coverage of HSR and the one-hour circle of high-speed rail in urban agglomeration is gradually forming. However, there is still a big gap between Beijing Tianjin Hebei and the Yangtze River Delta, Pearl River Delta. In addition, the economic coordinated development in Beijing-TianjinHebei is not as smooth as integrated transportation. Thus, HSR in Beijing-Tianjin-Hebei region is an essential part of the integration of the transportation network. This paper will take Beijing-Tianjin-Hebei area as an example to analyze the impact of the opening of high-speed railway on regional economic development.

The following section presents a literature review of the relationship between region economy and HSR. After that, the third section overviews the integrated development of Beijing-Tianjin-Hebei region. Then, the paper presents methods and data resource in the forth section, the fifth section presents results and analysis. Lastly, the sixth section concludes the paper. All in all, it is indisputable that convenient transportation will improve accessibility, reduce transportation cost and increase flow of other factors.

\section{Literature review}

With the continuous development of new economic geography, scholars have long acknowledged the relationship between transportation infrastructure and regional economy. The research is mainly related to growth pole theory and point-axis development theory. The connection between cities is inseparable from traffic, which in turn promotes the continuous optimization of regional spatial layout. In the $1950 \mathrm{~s}$, the economist Francois first proposed the growth pole theory, which is an unbalanced development idea. This theory agreed that developed regions with rich resources and high innovation capabilities, relying on own superiority, have achieved rapid economic growth and formed a growth pole. At the same time, developed areas have a radiation effect on 
surrounding areas through transportation infrastructure, thereby stimulating economic growth in the region. However, it will certainly lead to unbalanced economic development, which may be further deepened with the development of the growth pole. Chinese economic geographer Lu Dadao put forward the point axis theory, revealing the objective laws of regional spatial structure in the development, and providing a theoretical basis for the industries, cities and production layout along the transportation line.

Professors and scholars at home and abroad have conducted a lot of research. Kiyoushi highlights HSR has a positive impact on regional economy[1]. Sasaki studied the Shinkansen in Japan[2]. The opening of the Japanese Shinkansen improved accessibility, which in turn contributed to the improvement of the Japanese economy. José conducted a multi-level analysis around the country, region and locality. Through an empirical analysis of large and medium-sized cities along HSR, he clarified how the high-speed railway creates new opportunities for those large and medium-sized cities. Domestic scholars' research on HSR mainly focus on the change of traffic accessibility, the impact of city and regional economy and spatial organization structure. Zhang Xueliang et al. find that the construction of HSR will also bring negative spillover effects[3]. Lin Xiaoyan et al. studied the impact of the Beijing-Tianjin intercity high-speed rail on the city's economy[4]. The study find that the construction of HSR has gradually produced positive economic and social benefits. Sun Jiantao et al. show HSR has two effects on the regional economy[5]. One can be mainly manifested as industrial agglomeration effect, diffusion effect, same city effect and integration effect; another kind of influence is mainly manifested as siphon effect.

\section{Methods}

\subsection{Empirical model}

A quantitative single case-study approach is used in gaining economic gravitation between regions. The empirical model what we will use is developed based on the classical gravity model. The classical gravity model has been widely applied in the field of international trade in recent decades. Dingbergen firstly used it to explore the impact of regional economy on bilateral trade flow.

This paper aims to explore the changes of economic gravitation between regions before and after the opening of high-speed railway with the help of the improved gravitation model, which is shown in the following Equation (1). The following empirical model is considered with this formula(1).

$$
F_{\mathrm{ij}}=K \frac{\sqrt{P_{\mathrm{i}} G_{\mathrm{i}}} \sqrt{P_{\mathrm{j}} G_{\mathrm{j}}}}{D_{\mathrm{ij}}^{2}}
$$

Generally speaking, in the improved gravitation model, the dependent variable $F_{\mathrm{ij}}$ represents economic gravitation between two cities, $P_{\mathrm{i}}, P_{\mathrm{j}}$ respectively represent the urban permanent population and $G_{\mathrm{i}}, G_{\mathrm{j}}$ respectively represent the economic factors of the two cities. We often define $\sqrt{P_{\mathrm{i}} D_{\mathrm{i}}}, \sqrt{P_{\mathrm{j}} D_{\mathrm{j}}}$ as the urban quality of the city $\mathrm{i}$ and city j. $D_{\mathrm{ij}}$ represents the time distance between the two cities. $\mathrm{K}$ is the economic gravity coefficient, usually assumed to be 1 .

\subsection{Data resource}

This paper compares the statistics of 13 cities in BeijingTianjin-Hebei Urban agglomeration in 2011 and 2018, which consists of two municipalities of Beijing and Tianjin, as well as 11 prefecture level cities in Hebei Province, including Baoding, Tangshan, Shijiazhuang, Langfang, Qinhuangdao, Zhangjiakou, Chengde, Cangzhou, Hengshui, Xingtai, Handan. The process of coordinated development has been greatly accelerated after the opening of the Beijing-Shijiazhuang high-speed railway in 2012 . The study period considered in this paper is therefore between 2011 and 2018. A data set of 13 prefecture-level for this time period are extracted from the China Statistical Yearbook, the China City Statistical Yearbook, and the China Statistical Yearbook for Regional Economy.

\subsection{Data description}

Particularly, in view of the availability of data, we choose the shortest travel time between two cities when calculate the time distance before and after the opening of the highspeed rail. In this paper, the statistical data of the official website of the train ticket booking system are used to get the shortest running time between pairs in 13 cities of Beijing, Tianjin and Hebei. When round-trip-time between two cities is different, the smaller value shall prevail. If there is no direct high-speed railway between the two cities, it is assumed that there is no time cost of transfer to the destination through other cities, so the cumulative high-speed rail operation time between the two cities is used as the shortest travel time.

Now Suppose that the average speed of high-speed railway is $300 \mathrm{~km} / \mathrm{h}$, the average speed of expressway is $120 \mathrm{~km} / \mathrm{h}$, the average speed of railway is $90 \mathrm{~km} / \mathrm{h}$, and the ordinary highway is $80 \mathrm{~km} / \mathrm{h}$. Therefore, we get the shortest travel time between cities before and after the opening of high-speed rail. The shortest travel time after the opening of HSR is shown in Table 1.

Table1. The shortest travel time after the high-speedrailway is opened between pairs

\begin{tabular}{|c|c|c|c|c|c|c|c|c|c|c|c|c|}
\hline \multirow{2}{*}{$\begin{array}{c}\text { Time } \\
\text { (Minutes) }\end{array}$} & \multicolumn{12}{|c|}{ Cities } \\
\hline & BJ & $\mathrm{TJ}$ & SJZ & ZJK & $\mathrm{TS}$ & QHD & $\mathrm{CD}$ & $\mathrm{XT}$ & HD & HS & $\mathrm{CZ}$ & $\mathrm{BD}$ \\
\hline Tianjin & 38 & - & & & & & & & & & & \\
\hline Shijiazhuang & 70 & 91 & - & & & & & & & & & \\
\hline
\end{tabular}




\begin{tabular}{|c|c|c|c|c|c|c|c|c|c|c|c|c|}
\hline \multirow{2}{*}{$\begin{array}{c}\text { Time } \\
\text { (Minutes) }\end{array}$} & \multicolumn{12}{|c|}{ Cities } \\
\hline & BJ & $\mathrm{TJ}$ & SJZ & ZJK & TS & QHD & $\mathrm{CD}$ & XT & HD & HS & $\mathrm{CZ}$ & $\mathrm{BD}$ \\
\hline Zhangjiakou & 98 & 134 & 161 & - & & & & & & & & \\
\hline Tangshan & 64 & 40 & 117 & 165 & - & & & & & & & \\
\hline Qinhuangdao & 91 & 68 & 145 & 193 & 37 & - & & & & & & \\
\hline Chengde & 108 & 136 & 177 & 196 & 104 & 113 & - & & & & & \\
\hline Xingtai & 105 & 126 & 38 & 201 & 155 & 181 & 212 & - & & & & \\
\hline Handan & 110 & 131 & 43 & 206 & 160 & 186 & 217 & 26 & - & & & \\
\hline Hengshui & 114 & 107 & 75 & 210 & 133 & 159 & 221 & 89 & 95 & - & & \\
\hline Cangzhou & 59 & 43 & 106 & 160 & 69 & 95 & 163 & 142 & 147 & 80 & - & \\
\hline Baoding & 58 & 79 & 49 & 154 & 107 & 134 & 165 & 84 & 89 & 92 & 94 & - \\
\hline Langfang & 47 & 46 & 89 & 143 & 75 & 101 & 153 & 124 & 129 & 126 & 62 & 81 \\
\hline
\end{tabular}

\section{Results and analysis}

The panel data described in the preceding section is applied to the improved gravitation model. Then, the strength matrix of economic connection between two cities before and after the opening of high-speed railway can be calculated, as is shown in Table 3 and Table 4 . These results suggest that the economic connection intensity between cities has been greatly increased after the high-speed railway opened.

Table2. The economic linkage matrix before the opening of the high-speed rail in 2011

\begin{tabular}{|c|c|c|c|c|c|c|c|c|c|c|c|c|}
\hline \multirow{2}{*}{$\begin{array}{c}\text { Economic gravity } \\
\text { (Fij) }\end{array}$} & \multicolumn{9}{|c|}{ Cities } \\
\hline & BJ & TJ & SJZ & ZJK & TS & QHD & CD & XT & HD & HS & CZ & BD \\
\hline Tianjin & 5238.0 & - & & & & & & & & & & \\
\hline Shijiazhuang & 449.2 & 279.3 & - & & & & & & & & & \\
\hline Zhangjiakou & 415.1 & 105.4 & 29.7 & - & & & & & & & & \\
\hline Tangshan & 1091.4 & 1715.8 & 81.0 & 33.7 & - & & & & & & & \\
\hline Qinhuangdao & 132.1 & 109.2 & 13.0 & 5.9 & 184.6 & - & & & & & & \\
\hline Chengde & 302.0 & 100.5 & 17.4 & 11.3 & 108.3 & 27.4 & - & & & & & \\
\hline Xingtai & 116.5 & 78.4 & 378.0 & 8.3 & 27.0 & 4.9 & 5.8 & - & & & & \\
\hline Handan & 150.7 & 101.6 & 335.4 & 11.3 & 36.3 & 6.8 & 8.0 & 1251.0 & - & & & \\
\hline Hengshui & 172.1 & 128.3 & 209.0 & 8.2 & 35.6 & 5.5 & 6.3 & 81.2 & 57.8 & - & & \\
\hline Cangzhou & 598.2 & 1027.6 & 163.3 & 20.6 & 178.4 & 21.4 & 18.7 & 50.8 & 61.5 & 136.1 & - & \\
\hline Baoding & 1024.8 & 587.0 & 385.3 & 43.3 & 127.4 & 17.6 & 25.0 & 71.7 & 84.2 & 117.2 & 222.9 & - \\
\hline Langfang & 1840.2 & 1365.9 & 68.2 & 25.2 & 158.6 & 15.7 & 21.4 & 18.6 & 24.1 & 32.7 & 171.3 & 165.4 \\
\hline
\end{tabular}

Table3. The economic linkage matrix after the high-speed railway opened in 2018

\begin{tabular}{|c|c|c|c|c|c|c|c|c|c|c|c|c|}
\hline \multirow{2}{*}{$\begin{array}{c}\text { Economic } \\
\text { gravity (Fij) }\end{array}$} & \multicolumn{12}{|c|}{ Cities } \\
\hline & $\mathrm{BJ}$ & $\mathrm{TJ}$ & SJZ & ZJK & $\mathrm{TS}$ & QHD & $\mathrm{CD}$ & $\mathrm{XT}$ & HD & HS & $\mathrm{CZ}$ & $\mathrm{BD}$ \\
\hline Tianjin & 30313.6 & - & & & & & & & & & & \\
\hline Shijiazhuang & 4098.4 & 1625.2 & - & & & & & & & & & \\
\hline Zhangjiakou & 693.9 & 248.7 & 79.0 & - & & & & & & & & \\
\hline Tangshan & 4608.4 & 7906.5 & 424.0 & 70.7 & - & & & & & & & \\
\hline Qinhuangdao & 694.3 & 833.3 & 84.1 & 15.7 & 1213.8 & - & & & & & & \\
\hline Chengde & 501.2 & 211.8 & 57.4 & 15.5 & 156.2 & 21.1 & - & & & & & \\
\hline Xingtai & 919.8 & 428.1 & 2159.2 & 25.6 & 122.0 & 89.9 & 20.2 & - & & & & \\
\hline Handan & 1209.5 & 571.5 & 2433.7 & 35.2 & 165.2 & 121.7 & 27.8 & 3361.3 & - & & & \\
\hline Hengshui & 518.1 & 394.1 & 368.0 & 15.6 & 110.0 & 55.8 & 12.3 & 132.0 & 167.2 & - & & \\
\hline Cangzhou & 3856.6 & 4865.9 & 367.4 & 53.5 & 814.9 & 319.5 & 45.2 & 103.4 & 139.2 & 216.2 & - & \\
\hline Baoding & 4647.6 & 1678.9 & 2002.1 & 67.3 & 394.7 & 286.1 & 51.4 & 344.0 & 442.3 & 190.4 & 363.7 & - \\
\hline Langfang & 4381.8 & 3065.6 & 375.7 & 48.3 & 497.3 & 297.7 & 37.0 & 97.7 & 130.3 & 62.9 & 517.6 & 353.1 \\
\hline
\end{tabular}

\subsection{Economic connection intensity between cities before the opening of HSR}

From the overall point of view of the urban agglomeration, there is a huge gap that ranges from 4.9 to 5238.0 in the economic connection intensity between the two cities. Beijing and Tianjin, the two economic growth poles in the region, have weak economic connections with other cities in Hebei Province. The biggest economic 
gravitation value among cities is the Beijing-Tianjin city pair(5238.0), which is far superior to other city pairs thanks to the location advantage. However, the gravitation values of other cities are all below 1000. In addition, the cities pairs that are mostly located at the edge of the region or in a relatively remote location from the city center, generally have lower economic connections, such as Chengde-Xingtai(5.8) and Xingtai-Qinhuangdao(4.9) .

From the perspective of vertical comparison of core cities, Table 3 shows that not only is the economic radiation ability of the core cities in the urban agglomeration obviously insufficient, but the economic gravity intensity has an evident negative correlation with distance between core cities and others. The economic connections values of Beijing-Tangshan, BeijingLangfang, Beijing-Baoding, Tianjin-Langfang and Tianjin-Tangshan, are between 1000-2000, forming a close area of economic connections. Besides, Langfang has the closest connections with Beijing and Tianjin. On the one hand, Langfang can be effectively radiated by core cities because of the unique geographical advantage; on the other hand, it strives to break through the constraints of administrative divisions. Thus, Langfang is the first prefecture-level city in Hebei province which is driven by Beijing and Tianjin.

In summary, the economic connections generally formed a connection beam radiating from Beijing-Tianjin as the main sub-center to the surrounding circle. The economic connection intensity is directly related to the relative geographical location of cities. Therefore, Beijing, Tianjin, Tangshan and Langfang have close economic connections with surrounding cities by taking advantage of their concentrated location and convenient transportation. On the contrary, Qinhuangdao, Chengde, Zhangjiakou, Xingtai and Hengshui, which are located on the edge of the region, have the worst connection with other cities due to low accessibility before the high-speed rail is opened.

\subsection{Economic connection intensity between cities after the HSR is opened}

From the overall point of view of the urban agglomeration, after the opening of the HSR, the economic connection intensity between cities has been significantly strengthened, which is 4-7 times before the high-speed rail is opened. The economic connection intensity of the Beijing-Tianjin city pair has increased six times in the past seven years. At the same time, among cities is the BeijingTianjin city pair is the only one whose economic connections breaks 10,000 . Secondly, in addition to Beijing and Tianjin, the number of city pairs with economic connections above 1000 has increased to 16 . Lastly, the radiation effect of Beijing and Tianjin has been enhanced and the economy of the surrounding areas has been significantly driven. To sum up, it presents a pattern of gradually decreasing outwards with Beijing-Tianjin as the two levels, followed by Langfang, Baoding, and Tangshan.

From the perspective of vertical comparison of core cities, the disadvantage of geographic location marginalization has been alleviated, and the accessibility has shown a substantial increase due to the continuous improvement of traffic conditions. As a result, the economic connection intensity has multiplied. Specifically, compared with other cities in the province, Shijiazhuang, the provincial capital, has the closest economic connections with Beijing and Tianjin. Then, each city has the largest economic connection with Beijing, rather than cities which close to it, indicating that Beijing is indeed more attractive. What's more, cities in remote areas, such as Zhangjiakou and Chengde, have strengthened connections with other cities, but there are still huge differences in the economic connection intensity between cities. With the official opening of the Beijing-Zhangjiao HSR on December 30, 2019, the economic connection intensity between Zhangjiakou and Beijing-Tianjin will become increasingly close.

All in all, after the high-speed railway is opened, the trend has changed to be centered on the "Beijing-TianjinLangfang" line, gradually weakening toward the northsouth direction. The surrounding cities of Beijing-Tianjin have functions of receiving radiation and driving outwards. However, most of the small and medium-sized cities have faults in their economic connections with surrounding cities, due to the poor transportation infrastructure and lack of direct access to HSR.

\section{Conclusions}

To sum up, this study applied regional data to analyse the changes of economic gravitational values of 13 cities in the Beijing-Tianjin-Hebei urban agglomeration before and after the opening of the high-speed rail. The comparison of the two results reveals that the completion of the HSR will drive regional economic growth in the short term, especially China's economy has entered a period of new normal. Moreover, the correlation between traffic accessibility and economic connections has increased significantly, indicating that the impact of traffic on economic connection intensity has been apparently strengthened. It is also found that the intensity of regional spatial economic connections presents a pattern of gradually decreasing outwards with Beijing-Tianjin as the two levels before the opening of the high-speed railway and Beijing-Tianjin have a single polarization tendency. After the opening of the HSR, improved transportation infrastructure can effectively reduce transportation costs, increase factors mobility and improve regional accessibility. Thus, the region presents an obvious Coreperiphery structure. The study also identified that location factors have a major impact on economic connections.

Although the construction of high-speed rail has played an important role in promoting regional economic growth, it is acknowledged that regional economy is affected by other factors. This calls for extension of this study to other empirical model that can explore the specific influencing factors of regional economy, such as difference-in-differences model(DID). Furthermore, the findings also show some sensitivity to the sample data, the conclusions in short-term cannot replace long-term effects. Therefore future research can expand the time span of the 
sample data and combine its own development characteristics of urban agglomeration.

The paper further offers a perspective of how to promote regional economy. On the one hand, the government can strengthen the construction of transportation facilities for high-speed railways in the region. On the other hand, policy makers should break the barriers of administrative divisions, strengthen contacts, and build a sharing platform for resources, funds, talents and other elements. Only in this way, can the cities in the region share economic achievements.

\section{References}

1. Kiyoushi $\mathrm{K}$ and Makoto O, "The Growth of City Systems with High speed Railway Systems," The Annals of Regional Science. No. 31. vol. 1, pp. 3956, 1997.

2. Komei Sasaki, Tadahiro Ohashi and Asao Ando. "High-speed Rail Transit Impact on Regional Systems: Does the Shinkansen Contribute to Dispersion?". The Annals of Regional Science, No. 31. 31, pp. 77-98, 1997.

3. Zhang Xueliang and Nie Qingkai, "High-Speed Rail Construction and the Regional Economic Integration in China," Modern Urban Research. 6rd ed., vol. 2, pp.7-10, 2010.

4. Lin Xiaoyan, "Adaptability evaluation on comprehensive urban transportation system:based on data of 19 sub-provincial cities," Technology Economics. 30rd ed., vol. 12, pp.64-69, 2011.

5. Sun Jiantao, "Analysis on the Impact of High-speed Railway on Regional Economy". Beijing Jiaotong University. 2012.

6. Piet. Rietveld, "Infrastructure and spatial economic development," The Annals of Regional Science, No. 29, 1995, pp. 117-119.

7. Luo Shen and Lin Xiaoyan, "An Empirical Study on the Evaluation of the Impact of High Speed Rail on China," Inquiry into Economic Issues. vol. 11, pp.7478, 2013.

8. Zhang Xueliang, "Has Transport Infrastructure Promoted Regional Economic Growth?- With an Analysis of the Spatial Spillover Effects of Transport Infrastructure," Social Sciences in China. 34rd ed., vol. 2, pp.24-27, 2013.

9. Sun Bing. "Research on Innovation of Regional Management of Beijing-Tianjin-Hebei Coordinated Development," Social Sciences in China. Management World, 7rd ed., pp.172-173, 2016.

10. Zhang Anlin, Pu Zexu, Wang Min and Zeng Junwei, "The impacts of Beijing-Tianjin intercity high-speed railway on regional social and economic development," Technology \& Economy in Areas of Communications. No. 1. pp.14-18, 2013. 